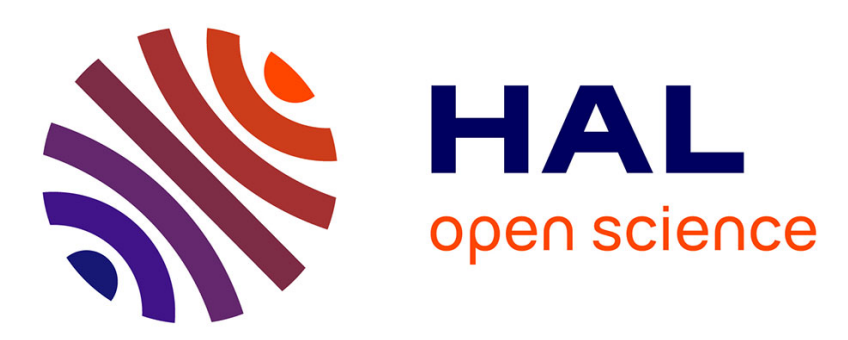

\title{
A 3D Split-Step Fourier Algorithm Based on an Discrete Spectral Representation of the Propagation Equation
}

\author{
Hang Zhou, Alexandre Chabory, Rémi Douvenot
}

\section{To cite this version:}

Hang Zhou, Alexandre Chabory, Rémi Douvenot. A 3D Split-Step Fourier Algorithm Based on an Discrete Spectral Representation of the Propagation Equation. IEEE Transactions on Antennas and Propagation, 2017, 65 (1988-1995), 10.1109/TAP.2017.2671018 . hal-01471666

\section{HAL Id: hal-01471666 \\ https://hal-enac.archives-ouvertes.fr/hal-01471666}

Submitted on 20 Feb 2017

HAL is a multi-disciplinary open access archive for the deposit and dissemination of scientific research documents, whether they are published or not. The documents may come from teaching and research institutions in France or abroad, or from public or private research centers.
L'archive ouverte pluridisciplinaire HAL, est destinée au dépôt et à la diffusion de documents scientifiques de niveau recherche, publiés ou non, émanant des établissements d'enseignement et de recherche français ou étrangers, des laboratoires publics ou privés. 


\title{
A 3D Split-Step Fourier Algorithm Based on a Discrete Spectral Representation of the Propagation Equation
}

\author{
Hang Zhou, Alexandre Chabory, and Rémi Douvenot
}

\begin{abstract}
A propagation method self-consistent in the discrete domain for the simulation of large distance scenarios in a 3D inhomogeneous atmosphere is proposed. First, a spectral representation of the field in a discrete 3D domain over an infinite perfect ground is introduced in cylindrical coordinates. A discrete spectral propagator is then derived. As in 2D methods, the inhomogeneous atmosphere is considered in the spatial domain using phase screens. An explicit numerical scheme is introduced. For directive sources, a sectoral propagation is proposed to reduce the computation time by limiting the computation domain. The method is tested by simulation scenarios involving refractive effects in both vertical and azimuthal directions. The 3D refractive effects are shown to be properly simulated.
\end{abstract}

Index Terms-Atmospheric propagation, discrete Fourier transforms, electromagnetic propagation, modeling, operators, parabolic wave equation, refraction.

\section{INTRODUCTION}

$\mathbf{T}$ HE parabolic equation method is an approximation of the wave equation valid along a paraxial direction neglecting backward propagation. This equation has been used extensively in radio wave propagation [1], e.g., for radar coverage prediction. One of the most efficient method for applying the parabolic equation is based on split-step Fourier methods as pioneered by Hardin and Tappert [2].

These algorithms can take into consideration the antenna pattern, the ground composition, the relief, and the atmosphere. Nevertheless, the backscattering is neglected. In order to evaluate the field propagation iteratively at increasing distances, the computation is realized going back and forth from a spatial to a spectral representation of the wave. Indeed, derivatives become multiplications in the spectral domain which simplifies and alleviates the computations.

The electrical characteristics of the atmosphere are taken into account in the spatial domain using phase screens [1]. The ground is taken into account by choosing a spectral transform consistent with the boundary condition. If the ground is a perfect electric conductor, the spectral transform corresponds to a sine or cosine Fourier transform depending on the considered field. For an impedance ground, the discrete mixed Fourier transform is usually used [3]. Note also that the ground relief can be accounted by means of a staircase approximation [1].

ENAC, TELECOM-EMA, F-31055 Toulouse, France,

Toulouse University, F-31400 Toulouse, France,

e-mail: hang.zhoulrecherche.enac.fr,

e-mail: chabory@recherche.enac.fr,

e-mail: douvenoterecherche.enac.fr.
These methods have initially been introduced for 2D cases, with either an axial symmetry in Cartesian coordinates or a rotational symmetry about the vertical axis in cylindrical coordinates. To model a $3 \mathrm{D}$ scene with the latter, a $\mathrm{N} \times 2 \mathrm{D}$ model approximation scanning all azimuth angles is classically used [2]. However, $\mathrm{N} \times 2 \mathrm{D}$ models neglect lateral effects. These latter can be significant, especially in the case of an irregular relief and/or non-constant refractive index.

In the context of underwater propagation of acoustic waves, 3D finite-difference or split-step Fourier methods have been used [4] [5] [6] [7]. The 3D models demonstrate a better accuracy than $\mathrm{N} \times 2 \mathrm{D}$ models where azimuthal couplings are present. The acoustic pressure and electromagnetic fields both satisfy the 3D Helmholtz equation, so the $3 \mathrm{D}$ propagation can be developed similarly for acoustic and electromagnetic propagation. Later on, for electromagnetic propagation, an extension of split-step methods based on the parabolic equation to 3D has been developed in Cartesian coordinates [8] [9].

The previous 3D methods are based on the parabolic equation, in which a paraxial approximation is applied to split the vertical and azimuthal derivative components. In this paper, this approximation is here bypassed by using an exact spectral representation of the field in a homogeneous atmosphere. This representation is obtained through a diagonalisation of the vertical operator as proposed by Chabory et al. [10]. Janaswamy has also derived a similar representation [11]. Besides, this operator has been extended to $3 \mathrm{D}$ in cylindrical coordinates in [12]. These methods are more accurate since no paraxial approximation is assumed. Indeed, they are derived from the Helmholtz equation, not from its parabolic approximated form.

Since the aforementioned methods are used for numerical simulations, the computed fields or potentials are expressed in the discrete domain. Therefore, a discrete formulation of the propagation equation is derived to achieve self-consistency according to the discrete electromagnetic theory [13]. The discrete mixed Fourier transform (DMFT) [3] has been a first effort towards this discrete formulation. Indeed, the boundary condition is given in the discrete domain by expressing the partial derivative along $z$ with a finite-difference approximation. However, the propagator is derived from the continuous spectral equation. This is an inconsistency in the DMFT theory.

In this paper, we fill the gap by deriving a 3D propagator from the discrete equations directly. Consequently, it is selfconsistent in the framework of the discrete electromagnetic theory. The approach is also valid in 2D. The ground and at- 


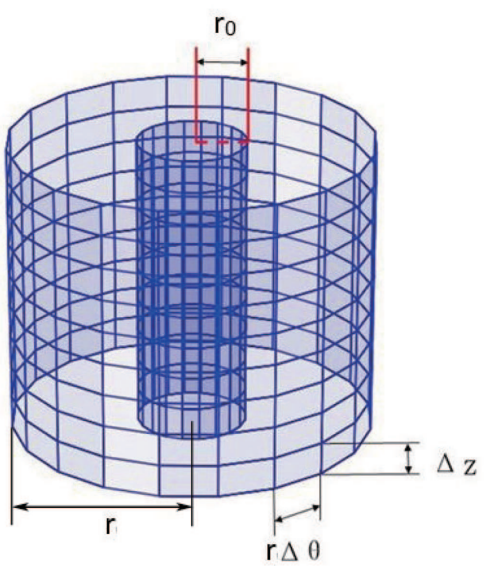

Fig. 1. Grids along $z$ and $\theta$ on the initial cylinder at the distance $r_{0}$ and on a cylinder at distance $r>r_{0}$.

mosphere effects are considered in the spatial domain as in 2D methods. For the sake of clarity, the theoretical development is given over a perfect metallic ground. Considering a slowly varying inhomogeneous atmosphere, the 3D split-step Fourier method is applied. Its explicit numerical scheme is introduced.

In Section 2, a homogeneous atmosphere is considered to express the problem in cylindrical coordinates by means of potentials. The propagation is expressed by means of the discrete spectral representation. The discretized form of the 3D formulation is presented. In Section 3, the explicit numerical scheme in an inhomogeneous atmosphere is obtained from phase screens and a 3D split-step algorithm. In Section 4, a sectoral propagation method is introduced. In Section 5, the sectoral propagation method and the 3D formulation are validated by comparison with an analytic expression. Finally, numerical tests are performed in the presence of inhomogeneous atmospheres to show the accuracy of the method.

\section{EXACT DISCRETE SPECTRAL REPRESENTATION IN A HOMOGENEOUS ATMOSPHERE}

\section{A. Configuration and continuous formulation}

We use the cylindrical coordinate system $(r, \theta, z)$ with unit vectors $(\hat{\boldsymbol{r}}, \hat{\boldsymbol{\theta}}, \hat{\boldsymbol{z}})$. We assume that the sources are located in the cylinder $r \leq r_{0}$ and that the fields are known at $r=r_{0}$. A perfect metallic and infinite planar ground is located at $z=0$. The propagation is computed in the region $r>r_{0}, z \geqslant 0$.

In a preliminary step, a homogeneous refractive index, i.e. $n$ constant, is considered and the vertical domain is limited to $z=z_{\max }$ where a PEC condition is applied. In this case, the fields can be decomposed in one transverse electric (TE) and one transverse magnetic (TM) components with respect to $z$ by means of Hertz potentials oriented along $\hat{z}$. For the electric field, omitting the time-dependence $e^{j \omega t}$ yields

$$
\boldsymbol{E}=k_{0}^{2} n^{2} \boldsymbol{\Pi}_{\mathrm{e}}+\nabla \boldsymbol{\nabla} \cdot \boldsymbol{\Pi}_{\mathrm{e}}-k_{0} \zeta_{0} n \boldsymbol{\nabla} \times \boldsymbol{\Pi}_{\mathrm{h}},
$$

where $\Pi_{\mathrm{e}}$ and $\boldsymbol{\Pi}_{\mathrm{h}}$ are the electric and magnetic vectorial potentials, $k_{0}$ is the wavenumber in free-space, and $\zeta_{0}$ is the free-space impedance.
Finally, replacing $\boldsymbol{\Pi}_{e}$ by $\frac{\Psi}{\sqrt{r}} \hat{z}$ gives the TM electric field

$$
\begin{aligned}
\boldsymbol{E}= & \frac{\partial}{\partial r}\left(r^{-\frac{1}{2}} \frac{\partial}{\partial z} \Psi\right) \hat{\boldsymbol{r}}+r^{-\frac{3}{2}} \frac{\partial^{2} \Psi}{\partial z \partial \theta} \hat{\boldsymbol{\theta}} \\
& +\left(r^{-\frac{1}{2}} \frac{\partial^{2} \Psi}{\partial z^{2}}+k_{0}^{2} n^{2} \Psi\right) \hat{\boldsymbol{z}}
\end{aligned}
$$

where $\Psi$ is the solution of

$$
\frac{\partial^{2} \Psi}{\partial r^{2}}+\frac{1}{r^{2}} \frac{\partial^{2} \Psi}{\partial \theta^{2}}+\frac{\partial^{2} \Psi}{\partial z^{2}}+\left(k_{0}^{2} n^{2}+\frac{1}{4 r^{2}}\right) \Psi=0 .
$$

Furthermore, due to the perfectly conducting walls at $z=0$ and $z=z_{\max }, \Psi$ fulfills the boundary conditions

$$
\begin{aligned}
& \left.\Psi\right|_{z=0}=0, \\
& \left.\Psi\right|_{z=z_{\max }}=0 .
\end{aligned}
$$

Note that the TE case can be formulated in a similar way from a $z$-directed magnetic potential $\Pi_{\mathrm{h}}=\frac{\Psi}{\sqrt{r}} \hat{z}$. The main differences are Neumann boundary conditions and a different expression for the electric field.

In the proposed method, the potentials are propagated. The electric field is obtained afterwards from the potentials.

\section{B. Discrete formulation}

For obvious numerical reasons, the computation domain is discretized and of finite size. Thus, the vertical domain is limited to $z \in] 0, z_{\max }$ [ and the following uniform grid is used

$$
\begin{array}{ll}
z=p_{z} \Delta z & \text { for } \quad p_{z}=\left\{1, \ldots, N_{z}-1\right\}, \\
\theta=p_{\theta} \Delta \theta & \text { for } \quad p_{\theta}=\left\{0, \ldots, N_{\theta}-1\right\}
\end{array}
$$

with $\Delta z=z_{\max } / N_{z}$ and $\Delta \theta=2 \pi / N_{\theta}$.

The grid is shown in Fig. 1. To obtain the discretized counterpart of (3), the second-order spatial derivatives with respect to $\theta$ and $z$ are discretized on the interior points of the grid using the central-difference approximation. This yields

$$
\frac{\partial^{2} \Psi}{\partial r^{2}}+\frac{1}{r^{2}} d_{\theta}^{2} \Psi+d_{z}^{2} \Psi+\left(k_{0}^{2} n^{2}+\frac{1}{4 r^{2}}\right) \Psi=0,
$$

with

$$
\begin{aligned}
& d_{z}^{2} \Psi_{p_{z}, p_{\theta}}=\frac{1}{\Delta z^{2}}\left(\Psi_{p_{z}+1, p_{\theta}}-2 \Psi_{p_{z}, p_{\theta}}+\Psi_{p_{z}-1, p_{\theta}}\right), \\
& d_{\theta}^{2} \Psi_{p_{z}, p_{\theta}}=\frac{1}{\Delta \theta^{2}}\left(\Psi_{p_{z}, p_{\theta}+1}-2 \Psi_{p_{z}, p_{\theta}}+\Psi_{p_{z}, p_{\theta}-1}\right),
\end{aligned}
$$

where $\Psi_{p_{z}, p_{\theta}}=\Psi\left(r, p_{\theta} \Delta \theta, p_{z} \Delta z\right)$.

\section{Discrete spectral representation}

To render our spectral representation numerically selfconsistent, we directly work from (6). Since Dirichlet and periodic boundary conditions are imposed along $z$ and $\theta$, the discrete spectral representation amounts to a discrete sine transform along $\hat{z}$ and a discrete Fourier transform along $\hat{\boldsymbol{\theta}}$. Hence, the spectral transform, denoted as $\tilde{\Psi}=U \Psi$, is explicitly given by

$$
\begin{aligned}
& \tilde{\Psi}_{q_{\theta}, q_{z}}(r)=\frac{1}{\sqrt{N_{\theta}}} \frac{1}{\sqrt{2\left(N_{z}+1\right)}} \sum_{p_{\theta}=0}^{N_{\theta}-1} \sum_{p_{z}=1}^{N_{z}-1} \Psi_{p_{\theta}, p_{z}}(r) \\
& e^{-j \frac{2 \pi p_{\theta} q_{\theta}}{N_{\theta}}} \sin \left(\pi \frac{p_{z} q_{z}}{N_{z}}\right) .
\end{aligned}
$$


Using fast trigonometric transforms, the computation of either $U \Psi$ or $U^{-1} \tilde{\Psi}$ can be efficiently performed, typically in $N_{\theta} N_{z} \log _{2} N_{\theta} N_{z}$ operations. Applying $U$ to (6) yields

$$
r^{2} \frac{\partial^{2} \tilde{\Psi}}{\partial r^{2}}+\left(r^{2} k_{r}^{2}-\kappa^{2}\right) \tilde{\Psi}+\frac{1}{4} \tilde{\Psi}=0
$$

where

$$
\kappa=\frac{2}{\Delta \theta} \sin \left(\frac{\pi q_{\theta}}{N_{\theta}}\right) \text { for } q_{\theta}=\left\{0, \ldots, N_{\theta}-1\right\},
$$

and $k_{r}^{2}=k_{0}^{2} n^{2}-k_{z}^{2}$ with

$$
k_{z}=\frac{2}{\Delta z} \sin \left(\frac{\pi q_{z}}{2 N_{z}}\right) \text { for } q_{z}=\left\{1, \ldots, N_{z}-1\right\} .
$$

Note that an impedance boundary condition could be considered by replacing the DST by the DMFT [14].

In a homogeneous atmosphere, the field can be propagated from $r_{0}$ to any distance $r>r_{0}$ in the spectral domain. Indeed, the analytical solution of (9) is [15]

$$
\tilde{\Psi}(r)=\frac{H_{\kappa}^{(2)}\left(k_{r} r\right)}{H_{\kappa}^{(2)}\left(k_{r} r_{0}\right)} \sqrt{\frac{r}{r_{0}}} \tilde{\Psi}\left(r_{0}\right),
$$

where $H_{\kappa}^{(2)}$ denotes the Hankel function of the second kind and order $\kappa$.

This equation is similar to the propagation of cylindrical harmonics except for the expressions of $k_{r}$ and $\kappa$ because of the discrete formulation. When $q_{\theta} \ll N_{\theta}$, i.e. for modes slowly varying with $\theta$, the order of the Hankel function becomes an integer $\left(\kappa \approx q_{\theta}\right)$ as in the continuous case [16].

For $\kappa$ is fixed and $\left|k_{r} r\right| \rightarrow \infty$, the Hankel function can be simplified to reduce the computation load. Doing so, the spectral propagation from $r_{0}$ to $r$ becomes

$$
\tilde{\Psi}(r) \approx \exp \left(-j k_{r}\left(r-r_{0}\right)\right) \tilde{\Psi}\left(r_{0}\right) .
$$

\section{EXPLICIT NUMERICAL SCHEME FOR A SLOWLY VARYING REFRACTIVE INDEX}

\section{A. Phase screens}

We aim at computing the effects of refraction in a 3D configuration in the presence of a planar and perfectly conducting ground. The atmosphere is characterized by a slowly varying refractive index $n$. To treat an inhomogeneous atmosphere, a method of phase screens similar to [3] is proposed.

From equation (3), we have

$$
\left[\frac{1}{k_{0}^{2}} \frac{\partial^{2}}{\partial r^{2}}+(A+B+1)\right] \Psi=0
$$

where

$$
\begin{aligned}
A & =\frac{1}{k_{0}^{2}}\left(\frac{1}{r^{2}} \frac{\partial^{2}}{\partial \theta^{2}}+\frac{\partial^{2}}{\partial z^{2}}+\frac{1}{4 r^{2}}\right), \\
B & =n^{2}-1 .
\end{aligned}
$$

The equation (14) is decomposed as a forward and a backward propagation terms. The backward term is neglected. Therefore, $\Psi$ fulfils

$$
\left(\frac{1}{k_{0}} \frac{\partial}{\partial r}-j \sqrt{A+B+1}\right) \Psi=0 .
$$

This formulation is exact for the forward propagation in a homogeneous atmosphere. In the case of a slowly varying refractive index, an approximation on the square root operator is necessary to end up with the phase screen method.

We split the operator by means of the approximation proposed by Feit and Fleck [14] that provides a wide-angle approximation. It is given by

$$
\sqrt{A+B+1} \approx \sqrt{1+A}+\sqrt{1+B}-1 .
$$

This is the basic of the split-step algorithm where the freespace propagator $\sqrt{1+A}$ is computed in the spectral domain while the effect of refraction $\sqrt{1+B}-1$ corresponds to a multiplication in the spatial domain by a phase screen. A higher-order approximation valid at wider angles can be applied [17].

\section{B. Numerical scheme}

To simulate the propagation, the domain $r \in\left[r_{0}, r_{\max }\right]$ is discretized in $N_{r}$ points, such that $r=r_{0}+p_{r} \Delta r$ for $p_{r} \in\left\{1, \ldots, N_{r}\right\}$. As with other split-step methods [1], the computation is performed marching on in distance. Between two consecutive cylinders, we propagate the potential through a homogeneous medium using the spectral propagator. Then we apply a phase screen to account for the refraction index variations.

At each iteration $p_{r}$, the propagation, the apodization and the local refractive index are considered using the 5 steps described below:

1) The potential $\Psi$ on the cylinder at distance $r_{0}+p_{r} \Delta r$ is expressed in the spectral domain by means of the transform $U$ defined by (8).

2) The spectrum is multiplied by the spectral operator $\Lambda_{p_{r}, q_{\theta}, q_{z}}$ representing the propagation from $p_{r} \Delta r$ to $\left(p_{r}+1\right) \Delta r$ given by

$$
\Lambda_{p_{r}, q_{\theta}, q_{z}}=\frac{H_{\kappa}^{(2)}\left(k_{r}(r+\Delta r)\right)}{H_{\kappa}^{(2)}\left(k_{r} r\right)} \sqrt{\frac{r+\Delta r}{r}},
$$

with $r=r_{0}+p_{r} \Delta r$.

3) The spectrum is expressed in the spatial domain with the transform $U^{-1}$.

4) To remove reflections over the top boundary, an apodization is applied with a Hanning window on the top half area in the spatial domain. This amounts to a term by term multiplication represented by a diagonal operator $W$.

5) A phase screen is applied which corresponds to a multiplication by

$$
R_{p_{r}, p_{\theta}, p_{z}}=\exp \left(j k_{0}\left(n_{p_{r}, p_{\theta}, p_{z}}-1\right) \Delta r\right),
$$

where $n_{p_{r}, p_{\theta}, p_{z}}$ is the refractive index at the position $\left(p_{r} \Delta r, p_{\theta} \Delta \theta, p_{z} \Delta z\right)$. The refraction term can be seen as a perturbation of the homogeneous case.

To conclude, the propagation from $p_{r} \Delta r$ to $\left(p_{r}+1\right) \Delta r$ is simulated step by step as

$$
\Psi_{p_{r}+1}=R W U^{-1} \Lambda U \Psi_{p_{r}}
$$

where $R, W$, and $\Lambda$ are diagonal operators, i.e. term-by-term multiplications. 


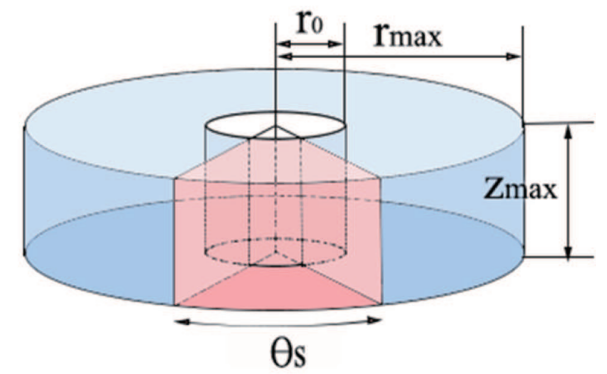

Fig. 2. Propagation sector of azimuthal width $\theta_{\mathrm{s}}=\frac{2 \pi}{N_{\mathrm{s}}}$ (in pink).

The final computation complexity of this method is of order $N_{r} N_{\theta} N_{z} \log _{2} N_{\theta} N_{z}$.

In the final step, the field is calculated from the potentials. To do so, the field spectrum is obtained from the potential spectrum by applying (2) in the spectral domain. Indeed, derivatives are easier to compute in the spectral domain. Finally, $U^{-1}$ is applied to obtain the fields.

\section{SECTORAL PROPAGATION}

When computing the propagation in a complex environment, two situations may occur. In the first one, you are only interested in the field within a limited angular width. In such a case, the most suitable solution in terms of computation time is to limit the computation domain to this angular sector and to impose absorbing boundary conditions [18]. In the second one, you want to compute the complete field, without truncating any energy. This is our aim here. Rather than simulating the propagation in the entire space with $\theta \in[0,2 \pi[$, the simulation can be limited to the sector where the power is located, denoted as the "propagation sector" thereafter. The azimuthal sector is chosen so that no energy reaches its boundaries.

Doing so, any kind of boundaries can be imposed. Periodic boundary conditions are used because they maintain the validity of the spectral Fourier representation, which renders its computation achievable by FFT.

A propagation sector of width $\theta_{\mathrm{s}}$ in azimuth is chosen. The calculation domain is represented in Fig 2. For the splitstep algorithm to remain unchanged, we impose $\theta_{\mathrm{s}}=\frac{2 \pi}{N_{\mathrm{s}}}$, where $N_{s}$ is an integer. Finally, the computation domain is $\theta \in\left[-\theta_{\mathrm{s}} / 2, \theta_{\mathrm{s}} / 2[\right.$ and $z \in] 0, z_{\max }[$.

If periodic boundary conditions are imposed at $\pm \frac{\theta_{s}}{2}$, the sectoral propagation is similar to the $2 \pi$-propagation except for a decrease of the angular period by a factor of $N_{\mathrm{s}}$. Thus, the truncation on $\theta$ amounts to a decimation of factor $N_{\mathrm{s}}$ in the spectral domain. This means a $N_{\mathrm{s}}$ times replication in the spatial domain as illustrated in Fig 3. To avoid signal overlap, the sector should be chosen such that no power goes through its boundaries. A numerical criteria is proposed in the test sections.

Finally, only a slight change in the previous formulation is necessary. The expression of the propagator (18) is kept, except for the order of the Hankel function $\kappa$ that becomes $\frac{2}{\Delta \theta} \sin \left(\frac{\pi N_{\mathrm{s}} q_{\theta}}{N_{\theta}}\right)$ for $q_{\theta}=\left\{0, \ldots, N_{\theta}-1\right\}$.

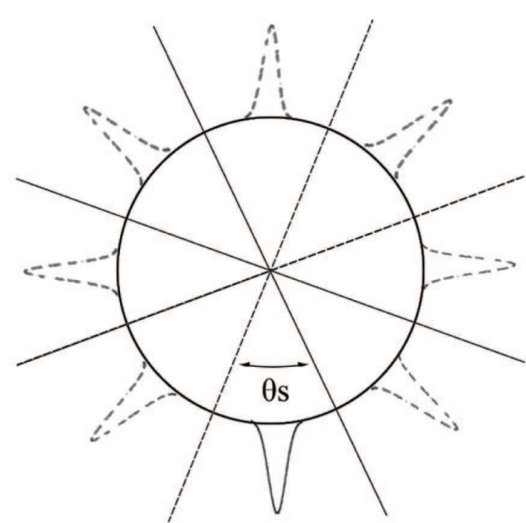

Fig. 3. Decimation of a factor $N_{\mathrm{s}}$ in the spectral domain amounts to a $N_{\mathrm{s}}$ times duplications in the spatial domain $\left(N_{\mathrm{s}}=8\right.$ in this figure).

\section{NUMERICAL TESTS OF THE 3D PROPAGATION}

The aims of the simulations are to test the sectoral propagation and to show that the proposed 3D method achieves to model vertical and azimuthal effects. On the contrary, $\mathrm{N} \times 2 \mathrm{D}$ methods cannot take the lateral effects into account.

In the simulations, the choice of the steps $\Delta z$ and $\Delta \theta$ depends on the Nyquist sampling theorem. The vertical and azimuthal samplings should be as [19]

$$
\Delta z \leq \frac{\lambda}{2 \sin \left(\alpha_{\max }\right)},
$$

and

$$
\Delta \theta \leq \frac{\lambda}{2 r_{\max } \sin \left(\alpha_{\max }\right)},
$$

where $\alpha_{\max }$ is the maximum divergence angle of the beam with respect to the propagation direction.

In the numerical simulations, the aim is to test the validity of the proposed 3D propagation method. For the sake of simplicity, we use a fixed azimuthal grid, even if this yields a large number of points in azimuth to satisfy the Nyquist condition at the final step, which are not necessary for the previous steps. Several schemes could be used to adapt the number of points in azimuth with range [19] [20]. These schemes could be applied to the proposed formulation to reduce the computation time.

\section{A. Validation of the sectoral propagation in free space}

In order to test the sectoral propagation, a complex point source [21] is propagated and compared to its analytic expression.

The frequency is $3 \mathrm{GHz}$. The complex source is located at $x_{s}=\frac{j k_{0} W_{0}^{2}}{2}, y_{s}=0 \mathrm{~m}, z_{s}=1000 \mathrm{~m}$, with $W_{0}=1 \mathrm{~m}$. The simulation parameters are: $r_{0}=2 \mathrm{~km}, r_{\max }=12 \mathrm{~km}$, $\Delta r=200 \mathrm{~m}, z_{\max }=2000 \mathrm{~m}, \Delta z=0.2 \mathrm{~m}$, and $N_{\theta}=1000$. The propagation is in free space.

Because a complex source corresponds to a Gaussian beam upon assuming the paraxial approximation, an angular sector of width $2 \arctan \left(\frac{2}{k_{0} W_{0}}\right)$ contains $88.47 \%$ of the beam power in the far field zone [22]. To guarantee that no power reaches 


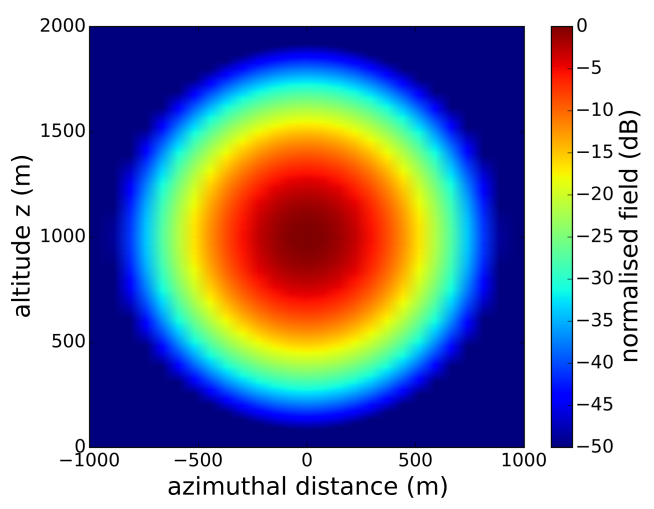

(a) Normalised electric field at the final step of the sectoral propagation

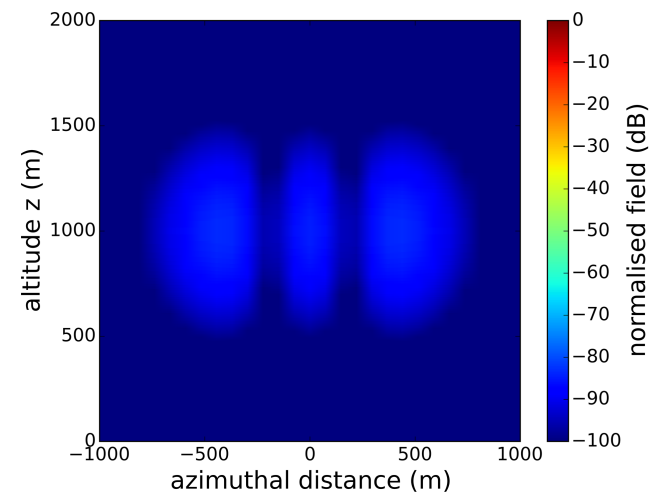

(b) Difference between the 3D sectoral propagation and the analytic solution

Fig. 4. Comparison between the 3D sectoral propagation and the analytic solution.

the boundaries, the sector is chosen 5 times larger. This yields $\theta_{s}=\frac{\pi}{10}$ and $N_{s}=20$.

The electric field at distance $r_{\max }$ computed with the sectoral propagation is shown in Fig 4(a). The difference between the sectoral propagation and the analytic solution is shown in Fig 4(b). The maximum difference is of order $-73 \mathrm{~dB}$. This difference is negligible. Therefore, the 3D sectoral propagation method is considered as successfully tested.

This simulation is performed on a standard desktop computer. The computation time of the sectoral propagation method is about 1.1 hours, whereas the total $2 \pi$ propagation spends about 21 hours. The computation time is thus significantly reduced.

\section{B. Test of a $45^{\circ}$-slanted linear refractivity index model}

The atmosphere effects are considered through the refractive index $n$ and the modified refractivity $M$ given by [23]

$$
M=(n-1) \times 10^{6}+\frac{z}{R_{\mathrm{E}}},
$$

where $z$ is the height above the Earth's surface and $R_{\mathrm{E}}$ is the Earth's radius.

The method is now applied on a 3D scenario with an inhomogeneous atmosphere characterized by a $45^{\circ}$-slanted linear refractivity index.
The configuration parameters are as follows: the frequency is $3 \mathrm{GHz}$. The complex source is at an altitude of $500 \mathrm{~m}$ with $W_{0}=3 \mathrm{~m}$. The modified refractivity $M$ varies linearly along the direction $\hat{\boldsymbol{v}}=\frac{1}{\sqrt{2}}(\hat{\boldsymbol{y}}+\hat{\boldsymbol{z}})$. The modified refractivity gradient along $\hat{\boldsymbol{v}}$ is $c=d M / d \boldsymbol{v}=1 \mathrm{M}$-unit $/ \mathrm{m}$. This value is chosen strong in order to obtain significant refraction effects, even at few kilometers.

The parameters of the simulation are as follows: $r_{0}=2 \mathrm{~km}$, $r_{\max }=12 \mathrm{~km}, \Delta r=500 \mathrm{~m}, z_{\max }=1000 \mathrm{~m}, \Delta z=0.2 \mathrm{~m}$, and $N_{\theta}=30000$.

The initial and final fields are plotted in Fig. 5. The beam center of the final field is shifted in both altitude and azimuth due to refraction effects. The shifts of the beam center in $z$ and $\theta$ are $35.81 \mathrm{~m}$ and $34.80 \mathrm{~m}$ respectively. According to ray theory [24], this shift is given by

$$
l_{\text {shift }}=\frac{\left(r_{\max }-r_{0}\right)^{2} c \times 10^{-6}}{2} .
$$

The numerical application yields $l_{\text {shift }}=35.36 \mathrm{~m}$.

The ray approximation and the simulated values in the 2 directions are the same within an acceptable error. Therefore, the effects of the inhomogeneous atmosphere on vertical and horizontal directions are properly simulated. Note that the displacement along $\theta$ could not be simulated by a $N \times 2 D$ method.

\section{Tests on a $2 D$ atmospheric duct}

A more complex scenario with 3D effects along both vertical and azimuthal directions is presented. We consider a refractive index model identical in both directions with 2 ducts along the vertical and azimuthal directions. Therefore, similar effects are expected along both directions.

The refraction index in the $z-\theta$ plane is obtained by $M=$ $\sqrt{M_{z} M_{\theta}}$, where $M_{z}$ and $M_{\theta}$ are functions associated with the modified refractivity index along $z$ and $\theta$.

In the vertical direction, we consider a surface-based duct, which is modelled by a trilinear function $M_{z}$, as illustrated in Fig 6.

The parameters are: $M_{0}=330$ M-units, $z_{b}=950 \mathrm{~m}$, $z_{t}=100 \mathrm{~m}, z_{\max }=2000 \mathrm{~m}$, gradients $c_{0}=0.118 \mathrm{M}$-units $/ \mathrm{m}$, $c_{2}=-1.0 \mathrm{M}$-units $/ \mathrm{m}$. To remove the discontinuities of the gradient that are not realistic, we smoothen the duct by using the moving averages method on 200 points. The resulting modified refractivity $M_{z}$ is shown in Fig 7(a).

In the azimuthal direction, we use the same model mapped into cylindrical coordinates, as shown in Fig 7(b). Such large index variation in azimuth are not realistic in the troposphere, but are used to compare the 3D effect in $\theta$ and $z$.

Finally, the modified refractivity index on the $z-\theta$ plane is obtained and the gradient of the refractivity index are shown in Fig 8 on one cylinder.

For the simulation, the frequency is $3 \mathrm{GHz}$. The complex source is at an altitude of $1000 \mathrm{~m}$ with $W_{0}=1 \mathrm{~m}$. The simulation parameters are: $r_{0}=2 \mathrm{~km}, r_{\max }=12 \mathrm{~km}, \Delta r=$ $500 \mathrm{~m}, z_{\max }=2000 \mathrm{~m}, \Delta z=0.2 \mathrm{~m}$, and $N_{\theta}=30000$.

Similar results along the 2 directions are expected since the ducts are the same along the 2 axes. 


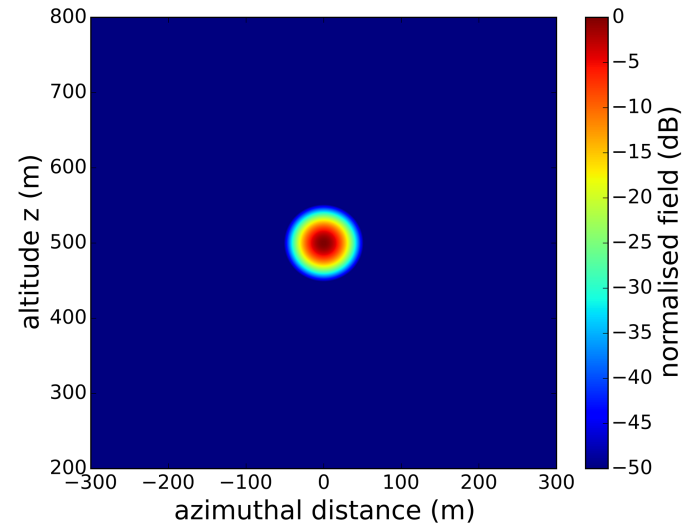

(a) Initial field

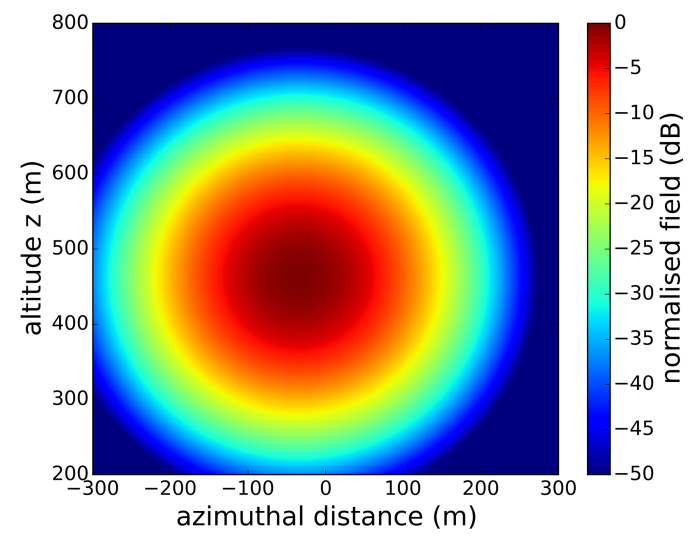

(b) Final field

Fig. 5. Initial and final normalised electric fields $(\mathrm{dB})$ about the direction of propagation.

The normalised electric field obtained at distance $r_{\max }=$ $12 \mathrm{~km}$ is shown in Fig 9. We can see the 3D effects due to the 2 ducts along the azimuthal and vertical directions. The maximum value of the final field is located at $63.0 \mathrm{~m}$ away from the centre on the vertical direction and at $62.8 \mathrm{~m}$ away on the azimuthal direction. The 2 values are the same within an acceptable error.

In Fig 10, we compare the electric field at $\pm 50 \mathrm{~m}$ away from the source beam center. The cuts correspond to the white dotted lines and solid lines in Fig 9. The plots match within $0.05 \mathrm{~dB}$. Therefore, the differences of the fields along the 2 directions are negligible. The effects due to the ducts are the same in both directions. The method is successfully tested since 3D complex effects are simulated.

For a comparison, the result of a $\mathrm{N} \times 2 \mathrm{D}$ method is shown in Fig 11. Only the vertical effects are accounted in this case.

\section{CONCLUSION}

The propagation method introduced in this work is based on a discrete spectral representation of the propagation equation related to both vertical and azimuthal variables in cylindrical coordinates.

Firstly, the discretised form of the 3D formulation has been presented. A homogeneous atmosphere has been considered to

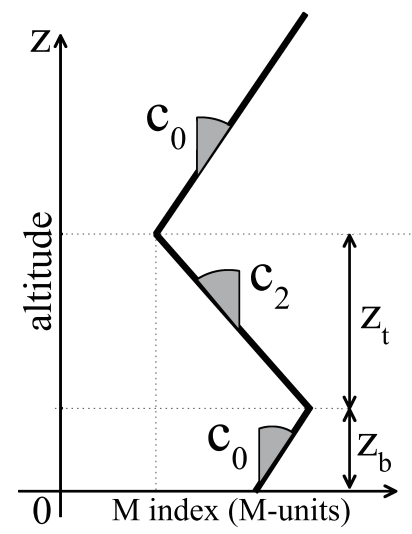

Fig. 6. Surface based duct trilinear model.

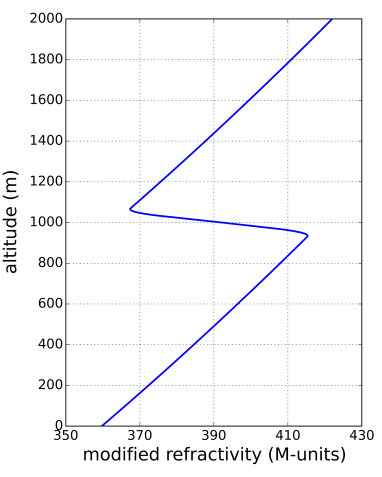

(a) Along $z$

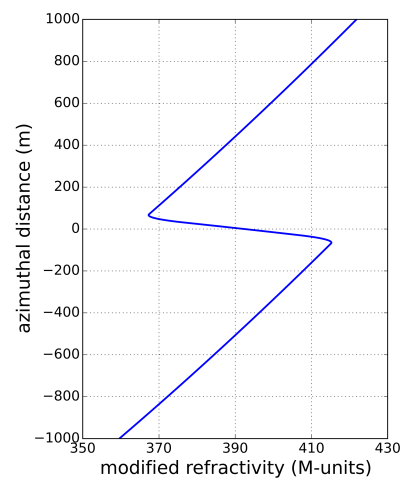

(b) Along $\theta$
Fig. 7. Trilinear refractivity models.

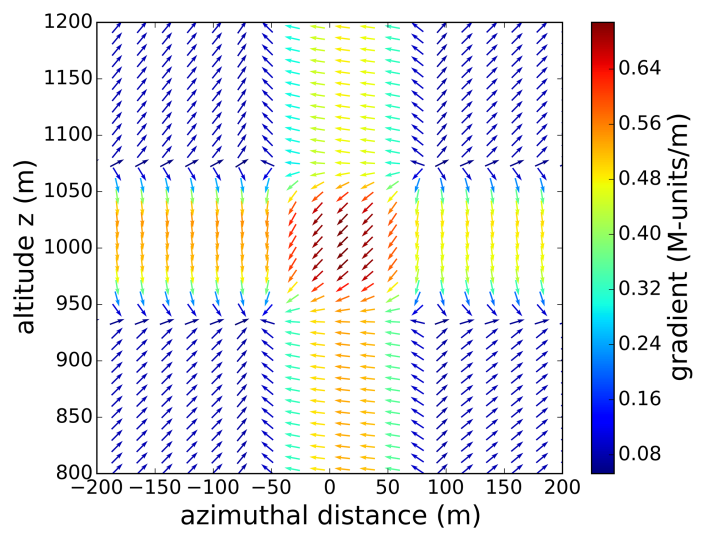

Fig. 8. Gradient vectors of the $\mathrm{M}$ index (M-units $/ \mathrm{m})$ on the $z-\theta$ plane. 


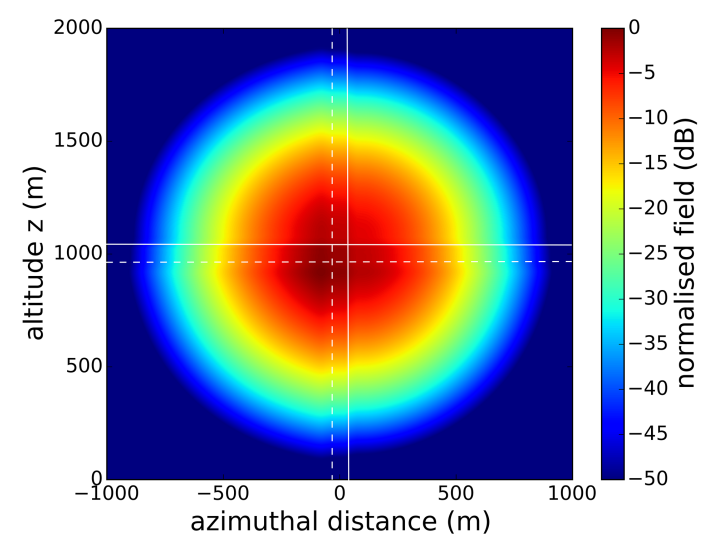

Fig. 9. Final field of the simulation using the proposed 3D method.

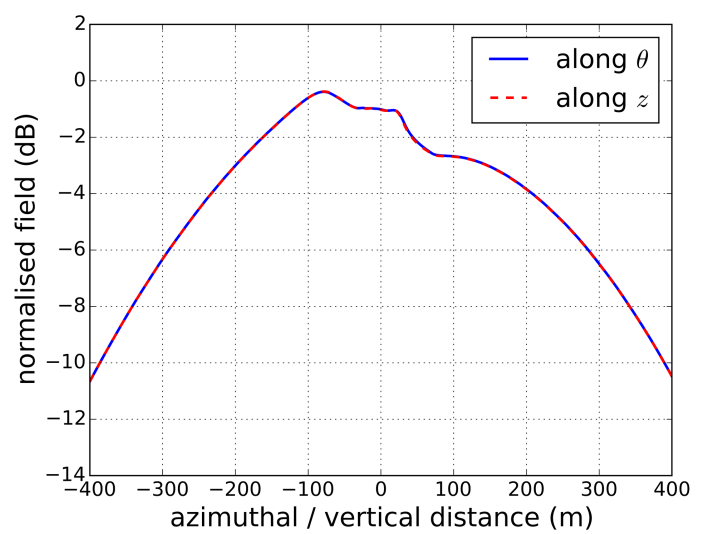

(a) Final fields along azimuthal direction at altitude $950 \mathrm{~m}$ (blue line) and along vertical direction at the distance $-50 \mathrm{~m}$ away on azimuthal direction from the center (red dotted line)

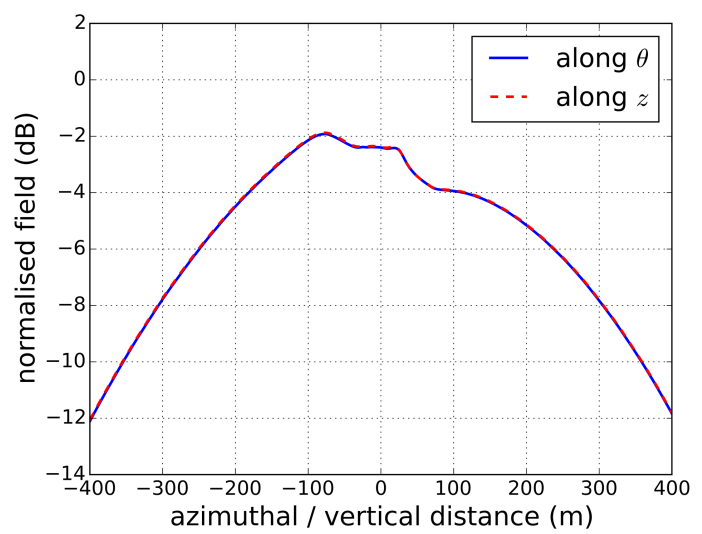

(b) Final fields along azimuthal direction at altitude $1050 \mathrm{~m}$ (blue line) and along vertical direction at the distance $50 \mathrm{~m}$ away on azimuthal direction from the center (red dotted line)

Fig. 10. Comparison of the fields along $z$ and $\theta$ directions.

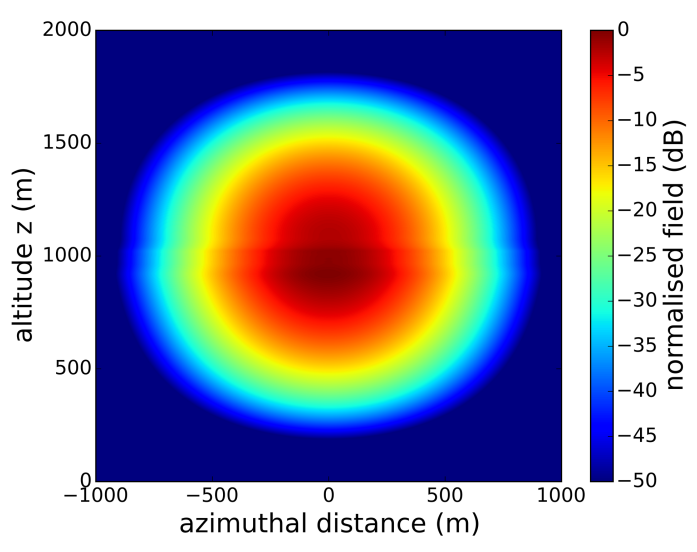

Fig. 11. Final field of the simulation using $\mathrm{N} \times 2 \mathrm{D}$ method.

express the problem by means of Hertz potentials. Then, the transform from the field to the spectrum has been explicitly given in discrete coordinates. A discrete spectral propagator has been derived.

Secondly, an explicit numerical scheme for considering a slowly varying refractivity has been introduced. The phase screen method valid at wide-angles is applied in the spatial domain. The propagation is considered with Hankel functions. An apodization is finally applied on the top half area to remove reflections over the top boundary.

In many cases, the source is directional. Therefore, a sectoral propagation method has been introduced. The propagation is modified to fit with the reduced computation domain. Depending on the angular size of the propagation sector, the computation time can be drastically reduced.

Finally, three numerical tests have been presented. Firstly, the sectoral propagation and the 3D formulation have been validated by a comparison with an analytical solution. Then the method has been applied in a 3D scenario with an inhomogeneous atmosphere characterized by a $45^{\circ}$-slanted linear refractivity index. The effects of the inhomogeneous atmosphere along vertical and horizontal directions are properly simulated. In the third simulation, a scenario with complex 3D effects along both vertical and azimuthal directions has been introduced. We have considered refractivity conditions with 2 atmospheric ducts along both the vertical and azimuthal directions. Note that the refractive effects have been successfully modelled along both directions. This method takes into account the azimuthal effects which is an advantage over $N \times 2 D$ models.

Numerical tests have proven that the simulation time is significantly reduced when using the sectoral propagation. This is a first step but other speed-up approaches are currently investigated. Besides, the relief and depolarization effects should be introduced. Also, confrontations with measurements in the presence of azimuthally inhomogeneous atmosphere could be considered.

\section{REFERENCES}

[1] M. Levy, Parabolic Equation Methods for Electromagnetic Wave Propagation. IET, 2000, no. 45. 
[2] R. H. Hardin and F. D. Tappert, "Applications of the split-step Fourier method to the numerical solution of nonlinear and variable coefficient wave equations," SIAM Review, vol. 15, no. 1, pp. 423-429, 1973.

[3] G. D. Dockery and J. R. Kuttler, "An improved impedance-boundary algorithm for Fourier split-step solutions of the parabolic wave equation," IEEE Transactions on Antennas and Propagation, vol. 44, no. 12, pp. 1592-1599, 1996

[4] W. L. Siegmann, G. A. Kriegsmann, and D. Lee, "A wide-angle threedimensional parabolic wave equation," Journal of the Acoustical Society of America, vol. 78, no. 2, pp. 659-664, 1985.

[5] F. Sturm and J. A. Fawcett, "On the use of higher-order azimuthal schemes in 3-D PE modeling," Journal of the Acoustical Society of America, vol. 113, no. 6, pp. 3134-3145, 2003.

[6] F. Sturm, "Numerical study of broadband sound pulse propagation in three-dimensional oceanic waveguides," Journal of the Acoustical Society of America, vol. 117, no. 3, pp. 1058-1079, 2005.

[7] J. A. Fawcett, "Modeling three-dimensional propagtion in an oceanic wedge using parabolic equation methods," J. Acoust. Soc. Am, 1993.

[8] A. A. Zaporozhets and M. F. Levy, "Bistatic RCS calculations with the vector parabolic equation method," IEEE Transactions on Antennas and Propagation, vol. 47, pp. 1688-1696, 1999.

[9] R. Janaswamy, "Path loss predictions in the presence of buildings on flat terrain: A 3-D vector parabolic equation approach," IEEE Transactions on Antennas and Propagation, vol. 51, no. 8, pp. 1716-1728, 2003.

[10] A. Chabory, C. Morlaas, R. Douvenot, and B. Souny, "An exact spectral representation of the wave equation for propagation over a terrain," in Electromagnetics in Advanced Applications (ICEAA), 2012 International Conference. IEEE, 2012, pp. 717-720.

[11] R. Janaswamy, "Radio wave propagation over a nonconstant immittance plane," Radio Science, vol. 36, no. 3, pp. 387-405, 2001.

[12] A. Chabory, C. Morlaas, and R. Douvenot, "An exact vectorial spectral representation of the wave equation for propagation over a terrain in 3D," in Antennas and Propagation in Wireless Communications (APWC), 2013 IEEE-APS Topical Conference, 2013, pp. 907-910.

[13] W. C. Chew, "Electromagnetic theory on a lattrice," J. Appl. Phys., vol. 75, no. 10, pp. 4843-4850, 1994.

[14] M. D. Feit and J. A. Fleck, "Light propagation in graded-index optical fibers," Applied Optics, vol. 17, no. 24, pp. 3990-3998, 1978.

[15] M. Abramowitz, Handbook of Mathematical Functions, M. Abramowitz and I. A. Stegun, Eds. New York: Dover Publications, Inc., 1966, vol. 55.

[16] R. F. Harrington, Time-Harmonic Electromagnetic Fields. Institute of Electrical \& Electronics Enginee, 2015.

[17] Y.-T. Lin and T. F. Duda, "A higher-order split-step Fourier parabolicequation sound propagation solution scheme," The Journal of the Acoustical Society of America, vol. 132, no. 2, pp. 61-67, 2012.

[18] F. B. Jensen, W. A. Kuperman, M. B. Porter, and H. Schmidt, Computational Ocean Acoustics. Modern Acoustics and Signal Processing, 2011.

[19] Y.-S. Lin, T. F. Duda, and A. E. Newhall, "Three-dimensional sound propagation models using the parabolic-equation approximation and the split-step Fourier method," Journal of Computational Acoustics, vol. 21, no. 01, p. 1250018, 2013.

[20] M. E. Austin and N. R. Chapman, "the use of tessellation in three dimensional parabolic equation modeling," J. Comp. Acous. 19, 221, 2011.

[21] G. A. Deschamps, "Gaussian beam as a bundle of complex rays," Electronics Letters, vol. 7, no. 23, pp. 684-685, November 1971.

[22] P. F. Goldsmith, Quasioptical Systems: Gaussian Beam Quasioptical Propagation and Applications. IEEE press New York, 1998.

[23] C. L. Pekeris, "Accuracy of the earth-flattening approximation in the theory of microwave propagation," Physical Review, vol. 70, no. 7-8, p. 518,1946

[24] Y. A. Kravtsov and Y. I. Orlov, Geometrical Optics of Inhomogeneous Media. Springer-Verlag, 1990

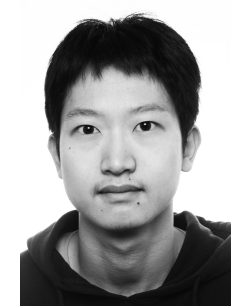

Hang Zhou was born in Tianjin, China, in 1990. $\mathrm{He}$ received the B.S. degree in aerodynamic from the University of Civil Aviation of China, Tianjin, China, in 2012 and the engineer degree in aerospace electronic from the Ecole Nationale de l'aviation civile, Toulouse, France, in 2014. He is a Ph.D. student in the group EMA, laboratory TELECOM in the Ecole Nationale de l'Aviation Civile, Toulouse, France, since 2015 .

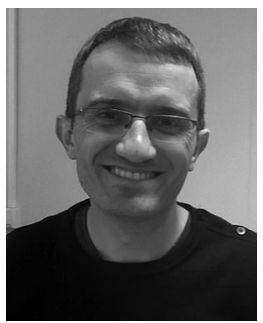

Alexandre Chabory Alexandre Chabory received the M.Sc. degree in electronic engineering from the French Civil Aviation University (ENAC), Toulouse, France, in 2001. He received the Ph.D. degree in electromagnetics from Paul Sabatier University, Toulouse, France, in 2004.

From 2004 to 2007, he was a Postdoctoral Scientist with the Eindhoven University of Technology (TU/e), Eindhoven, The Netherlands. Since 2007, he has been an Assistant Professor with the Electromagnetics and Antennas Research Group, Telecom Laboratory, ENAC, Toulouse, France. Since 2012, he has been the head of this group. His research interests include electromagnetic theory and modeling, mainly for aeronautical applications.

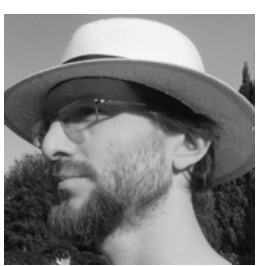

Rémi Douvenot was born in Chartres, France, in 1982. He received the engineer degree from the ENAC (French National Civil Aviation School), the M.Sc. from Toulouse University, France, in 2005, and the Ph.D. degrees in electrical engineering from Nantes University, France, in 2008. His Ph.D. was on refractivity from clutter. In 2009, he worked with the Laboratoire des Signaux et Systèmes (L2S), UMR (SUPELEC - CNRS - Univ Paris-Sud) on non-destructive testing using eddy-currents. Since 2011, he has been a lecturer in electromagnetics with TELECOM/EMA research group, ENAC in Toulouse, France. His main research interests are the theory of wave propagation and its applications to radar and air transport systems. 